\title{
Research Article \\ Photostability of Uranine via Crossed-Beam Thermal Lens Technique
}

\author{
M. Zein El-Din, ${ }^{1}$ K. Elsayed, ${ }^{2}$ S. M. Al-Sherbini, ${ }^{1}$ and M. A. Harith ${ }^{1}$ \\ ${ }^{1}$ Department of Laser Applications in Metrology, Photochemistry, and Agriculture, National Institute of Laser \\ Enhanced Sciences (NILES), Cairo University, Giza 12613, Egypt \\ ${ }^{2}$ Department of Physics, Faculty of Science, Cairo University, Giza 12613, Egypt
}

Received 2 October 2006; Revised 9 February 2007; Accepted 22 February 2007

Recommended by Savas Georgiou

\begin{abstract}
Uranine is a diagnostic aid in ophthalmology and is used as an immunohistological stain. A photostability study on this important compound using a crossed-beam thermal lens (TL) technique was carried out. The study is based on the photodegradation (PD) behavior and rate regarding some experimental parameters such as the incident laser power, wavelength, modulation frequency, and sample concentration. The effects of such parameters on the TL signal and PD rate are discussed in details. It has been found that the PD rate is proportional to the power of the pumping laser and the concentration of the sample within the investigated range. However, the modulation frequency is found not to influence the PD rate. The photochemical quantum yield has been measured using potassium ferrioxalate actinometry and it was found to be very low.
\end{abstract}

Copyright (c) 2007 M. Zein El-Din et al. This is an open access article distributed under the Creative Commons Attribution License, which permits unrestricted use, distribution, and reproduction in any medium, provided the original work is properly cited.

\section{INTRODUCTION}

Knowledge about drug photostability is vital from the medical point of view. This phenomenon is defined as the lightinduced loss of potency of a drug product that may lead to a therapeutically inactive drug. In turn, this may also lead to adverse effects due to the formation of minor degradation products during storage and administration [1]. In addition, the drug can cause light-induced side effects after administration to the patient by interaction with endogenous substances.

Sodium fluorescein, resorcinol phthalein sodium or uranine, is a highly fluorescent chemical compound. It absorbs visible light in the blue range with peak absorption and excitation band occurring at wavelengths between 465 and $490 \mathrm{~nm}$. The fluorescence emission occurs in the yellowgreen range from 520 to $530 \mathrm{~nm}$. These fluorescent properties have made fluorescein useful in a variety of medical applications such as ophthalmologic diagnostic (e.g., corneal trauma indicator, ophthalmic angiography, contact lens fitting) in the form of strips and intravenous injection to determine circulation time $[2,3]$.

In 1965, the photothermal lens (PTL) effect has been discovered by Gordon et al. [4] when they observed tran- sient power and beam divergence changes in the output of an He-Ne laser. Because of its high sensitivity and versatility, this technique has been widely used in spectroscopy, microvolume trace analysis and photodegradation, as well as thermo-optical characterization of solid, liquid, and gas samples [5-7].

The PTL results from optical absorption and heating of the sample in region localized to the extent of the excitation source. This lens-like element arises from the temperature dependence of the sample-refractive index. Moreover, because of the fact that most materials expand upon heating and the refractive index is proportional to the density, this lens usually has a negative focal length. This negative lens causes beam divergence and the signal is detected as a timedependent decrease in power at the center of the beam [8]. The technique has been recently developed to be exploited in other tasks such as the determination of fluorescence quantum efficiency and thermo-optical coefficients of fluorescent materials [9].

The present study is intended to investigate the photostability, based on photodegradation (photobleaching) behavior, of sodium fluorescein in its aqueous solution using a crossed-beam TL setup. 


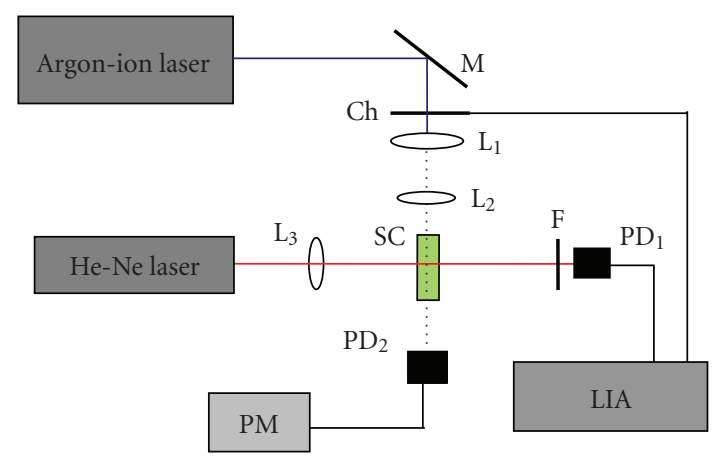

FIgURE 1: Schematic diagram of crossed-beam TL spectrometer, where $L_{2}$ and $L_{3}$ are convex lenses and $L_{1}$ is a cylindrical lens, SC is the sample cell, $\mathrm{F}$ is an interference filter, $\mathrm{PD}_{1,2}$ are photodiodes, $\mathrm{PM}$ is the power meter, $\mathrm{Ch}$ is a chopper, $\mathrm{M}$ is a mirror, and LIA is a lock-in amplifier.

\section{MATERIAL AND METHODS}

$10^{-3} \mathrm{~mol} / \mathrm{L}$ stock solutions of sodium fluorescein (uranine) puriss (Fluka Chemie GmbH, Taufkirirchen, Germany) were prepared in double distilled water. Different concentrations were prepared to study the photodegradation. The photochemical quantum yield was determined using potassium ferrioxalate, 1,10-phenanthroline, and sodium acetate buffer solution, puriss (FLUKA) by the conventional ferrioxalate actinometry as described by Hachard and Parker [10], and also by Murov [11]. UV-visible absorption measurements were carried out using spectrophotometer (Perkin Elmer Lambda 40). An argon-ion laser (Coherent Innova 400) with a wavelength of $488 \mathrm{~nm}$ was used to irradiate the sample within a $(3 \times 1 \times 1 \mathrm{~cm})$ quartz cuvette.

The experimental setup of the crossed-beam thermal lens employed in the photostability study is shown in Figure 1. The 488 and $514 \mathrm{~nm}$ excitation wavelengths from the argonion laser were used as pump sources to generate the TL in the investigated medium. The beam diameter was $2 \mathrm{~mm}$ before focusing and $400 \mu \mathrm{m}$ in the focusing case. An He-Ne laser light of wavelength $632.8 \mathrm{~nm}$ (Melles Griot, $10 \mathrm{~mW}$ ) was used as the probe beam. The pump beam is intensity modulated at different frequencies using a mechanical chopper (SR-540). The two laser beams are focused into the sample cell and strike it perpendicularly, an interference filter was placed in the path of the probe beam which allows only the $632.8 \mathrm{~nm}$ wavelength to reach the photodiode detector. The detected signal was then processed using a dual-phase lock-in amplifier (Stanford Research Systems SR-530) and was displayed on a CRO (not shown in Figure 1).

\section{RESULTS AND DISCUSSION}

Aquous solution of uranine gives a relatively low TL signal compared with other solvents. This is mainly due to its small change in the temperature refractive index $(d n / d T)$, besides its high thermal conductivity (K). However, it has been

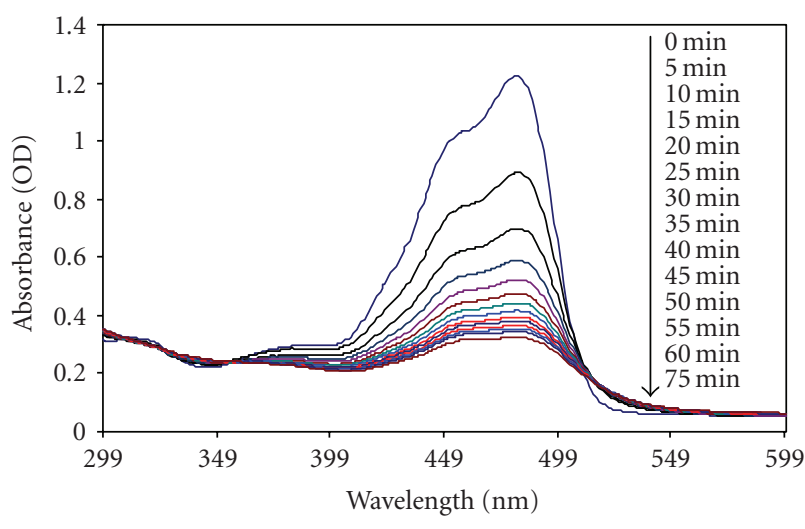

FIGURE 2: The absorption spectra of irradiated sodium fluorescein by argon laser light of $\lambda=488 \mathrm{~nm}$ at different exposure time intervals and constant sample concentration of $133 \times 10^{-6} \mathrm{~mol} / \mathrm{L}$.

decided to use aquous solutions throughout the performed measurements to simulate the conditions of the medical use of such substance where water is normally the most used solvent. Another reason is that water belongs to the class of nonabsorbing liquids. These liquids are highly transparent in the visible region and their thermal properties are well known. Therefore, the absorbed light energy is then due to the dissolved substance only [12].

Figure 2 shows the absorption spectra of $133 \times$ $10^{-6} \mathrm{~mol} / \mathrm{L}$ of sodium fluorescein irradiated by the $488 \mathrm{~nm}$ output of the argon laser. The figure demonstrates significant decreases in the optical density with increased exposure time without any changes in the maximum absorption band $\left(\lambda_{\max }=481 \mathrm{~nm}\right)$. The decrease in the optical density at the maximum wavelength may be due to the photodegradation of the uranine dye.

The photochemical quantum yield $\left(\phi_{c}\right)$ of uranine was measured using the potassium ferrioxalate actinometry method $[10,11]$ for a sample irradiated by argon laser light of $\lambda=488 \mathrm{~nm}$. Some constants used are as follows: the photochemical quantum yield $(\phi)$ of actinometer is 1.08 (at $488 \mathrm{~nm}, .0816 \mathrm{~mol} / \mathrm{L}, 23^{\circ} \mathrm{C}$ ) and the excitation coefficient of ferrous 1,10 -phenanthroline complex at $510 \mathrm{~nm}$ is $\left(\sim 1.11 \times 10^{4} \mathrm{l} / \mathrm{mol} . \mathrm{cm}\right)$. The obtained photochemical quantum yield was $2.2 \times 10^{-5}$.

The photostability based on the photodegradation (PD) behavior and the rate regarding some parameters will be discussed according to the following.

\subsection{Effect of the concentration}

Figure 3 shows the concentration dependence of the photodegradation $(\mathrm{PD})$ rate. The trends obtained are completely different from similar measurements reported on doped polymers (methyl methacrylate PMMA) exposed to dye laser light [7] which show inverse proportionality of PD rate with concentration. This discrepancy can be ascribed mainly to the different host matrix, whether liquid (in our case) or solid (in the polymer measurements). The absorbing species in 


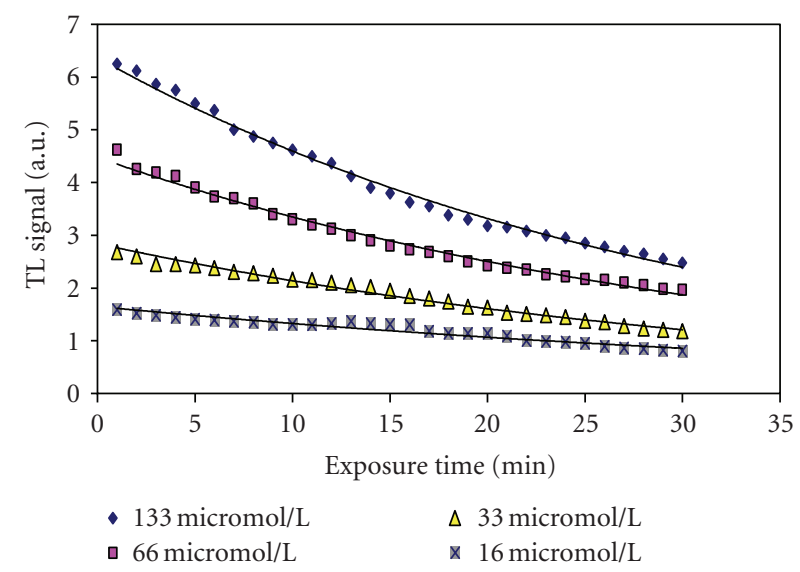

FIGURE 3: PD behavior of different concentrations represented in TL signal.

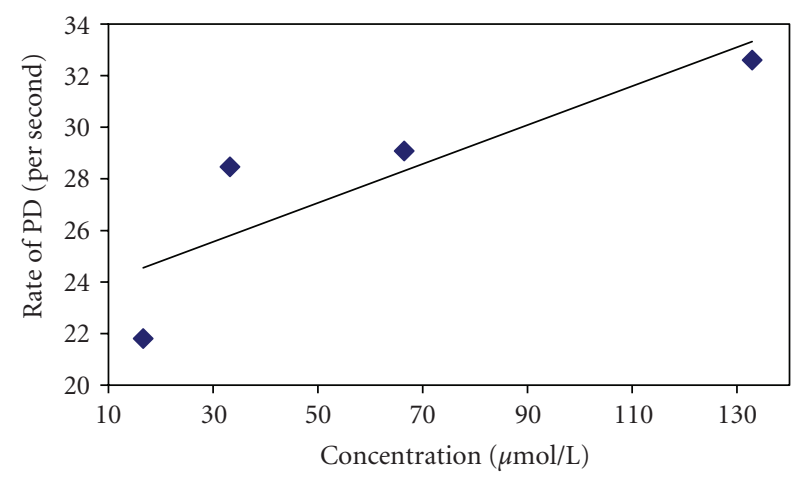

FIGURE 4: Variation of the rate of PD of Na-fluorescein with concentration.

the laser-exposed area will not be replaced after degradation due to the static nature of such species in case of a solid matrix. Consequently, the low concentration levels are logically faster in PD than the high levels. On the other hand, in case of solutions, the species in the exposed area are dynamic in laminar flow due to laser-induced heating, that is, undegraded molecules replace the degraded ones. Thus at higher concentrations, the released heat will be greater than that in the case of lower concentrations which can be observed evidently in the drop of the PD rate at lower concentration $(16 \mu \mathrm{mol} / \mathrm{L})$, see Figure 4 . Therefore, the convection currents will be higher leading to an increase in the vertical laminar flow of the solution within the sample cell, this in turn increases the PD rate of the molecules.

Slight differences between the rates of PD over the selected levels of concentration can be clearly observed as shown in Figure 4. The PD rate at a concentration of $133 \mu \mathrm{mol} / \mathrm{L}$ is found to be 1.12 times greater than that at a concentration of $66 \mu \mathrm{mol} / \mathrm{L}, 1.15$ times greater than that at a concentration of $33 \times 10^{-6} \mathrm{~mol} / \mathrm{L}$ and 1.5 times greater than that at a concentration of $16 \mu \mathrm{mol} / \mathrm{L}$.

\subsection{Effect of the pumping laser power}

The variation of $\mathrm{PD}$ rate with the power of the pumping laser is investigated at four power levels namely $220,320,420$, and $520 \mathrm{~mW}$. The other experimental parameters were fixed at $133 \mu \mathrm{mol} / \mathrm{L}$ sample concentration, $1.0 \mathrm{kHz}$ modulation frequency, and $488 \mathrm{~nm}$ laser wavelength. The observed variations in the thermal lens signal with time for such power levels shown in Figures 5(a) and 5(b). This reveals a clear proportionality between the incident power and PD rate. Furthermore, the increase in laser power is accompanied with an enhancement in the TL signal. These results are in a good agreement with that of Kurian et al. [7].

\subsection{Effect of the pumping laser wavelengths}

The effect of the pumping wavelength on PD rate and TL signal is studied adopting two different lines of argon ion laser at 488 and $514 \mathrm{~nm}$. The measurements are carried out while the other parameters are kept constant at $220 \mathrm{~mW}$ laser output power, $133 \mu \mathrm{mol} / \mathrm{L}$ sample concentration and $1 \mathrm{kHz}$ chopper frequency.

From the absorption spectra of Na-fluorescein Figure 2, it is clear that its absorption band falls in the blue-green region peaking at $489 \mathrm{~nm}$, which is very close to the argon laser blue line at $488 \mathrm{~nm}$ and far from the $514 \mathrm{~nm}$ line.

So, the PD rate (the slope of the tangential to the curve) in Figure 6 at $488 \mathrm{~nm}$ is higher by about 3.3 times than that at $514 \mathrm{~nm}$. In addition, the extreme signal of $488 \mathrm{~nm}$ higher than that of $514 \mathrm{~nm}$ is due to relative higher absorbance of Na-fluorescein at $488 \mathrm{~nm}$ than at $514 \mathrm{~nm}$ as shown in Figure 7. Consequently, it is clear that the pumping laser frequency has a major effect on PD rate and TL signal of Nafluorescein substance.

\subsection{Effect of the chopper frequency}

The effect of modulation frequency of the pumping laser beam on the PD rate and TL signal of Na-fluorescein is investigated at five distinct chopping frequencies: $500 \mathrm{~Hz}, 750 \mathrm{~Hz}$, $1 \mathrm{kHz}, 1.25 \mathrm{kHz}$, and $1.5 \mathrm{kHz}$. Such measurements are carried out at the same incident laser wavelength $(488 \mathrm{~nm})$ and power $(220 \mathrm{~mW})$, as well as the solution concentration $(133 \mu \mathrm{mol} / \mathrm{L})$. Figure 8 illustrates that within the range selected, the modulation frequency of the pump laser has no obvious effect on the PD rate of the sample molecules. This denotes that the PD process of Na-fluorescein depends only on the total incident energy per unit time on the sample, which is the same for all the chopping frequencies [13]. On the other hand, as predicted, TL signal has inverse proportionality with the modulation frequency as shown in Figure 9.

\section{CONCLUSION}

The dual-beam thermal lens technique has been found to be useful to study the photostability of sodium fluorescein in aqueous solution using a crossed-beam technique. The 


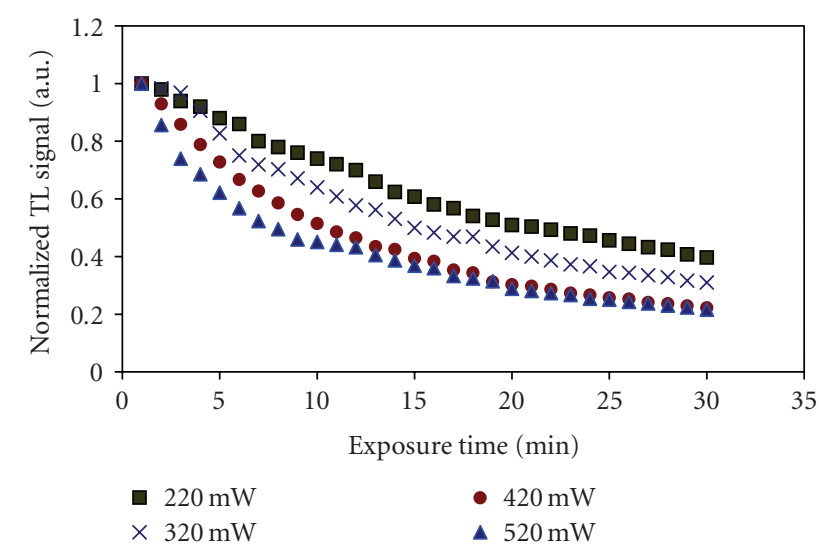

(a)

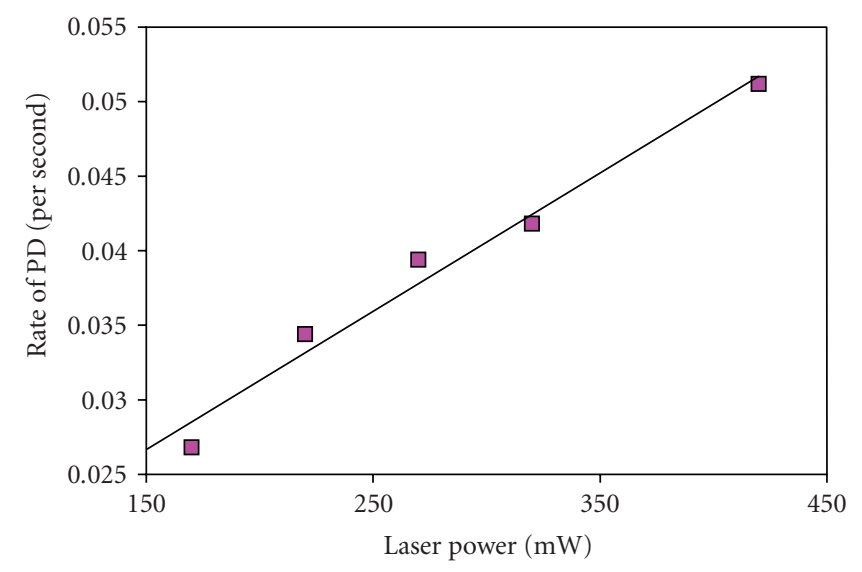

(b)

FIGURE 5: Variation of the rate of photodegradation with the incident laser power over 30-minute exposure time (a) and the PD rate change with the incident laser power (b).

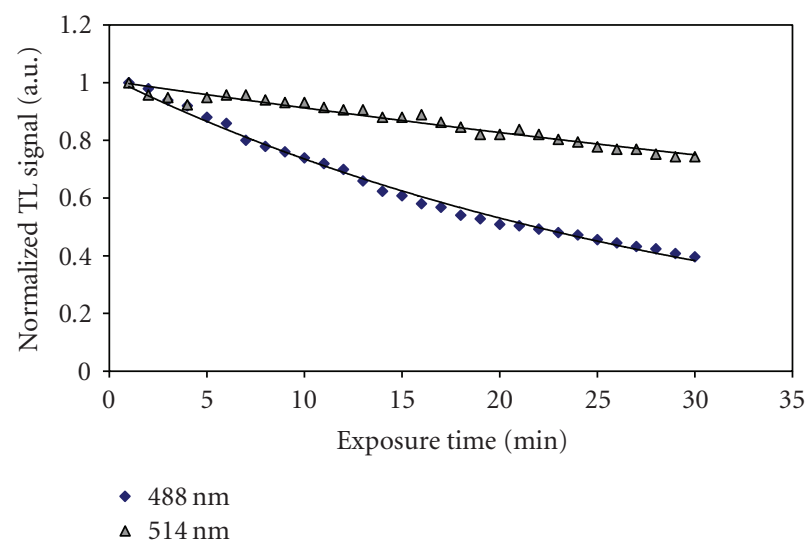

FIGURE 6: Variation of PD behavior of Na-fluorescein with wavelength.

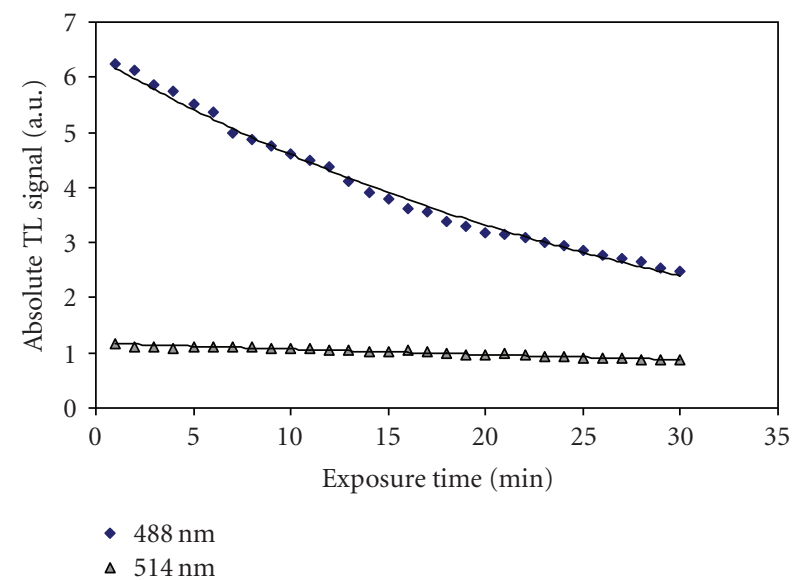

FIgURE 7: Variation of TL absolute signal of Na-fluorescein with wavelength of pump laser.
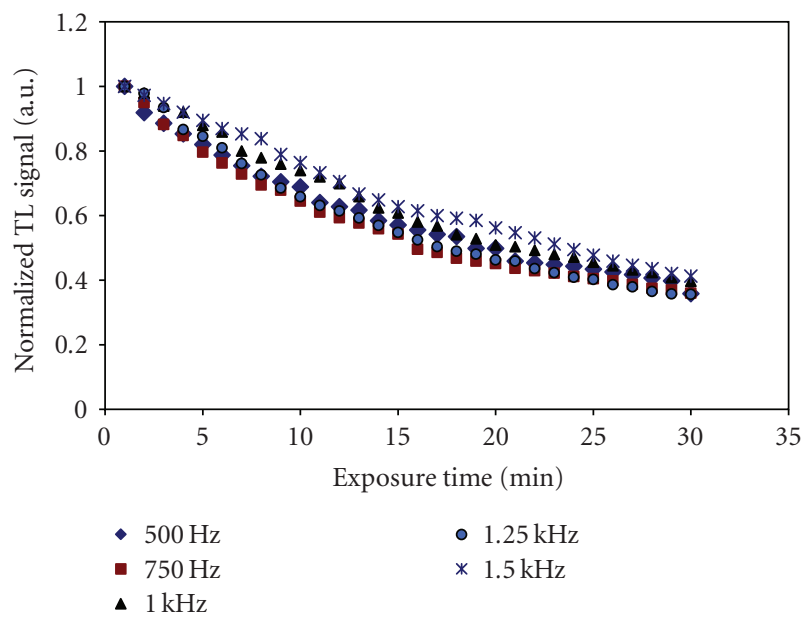

Figure 8: Effect of modulation frequency on PD rate of $\mathrm{Na}$ fluorescein.

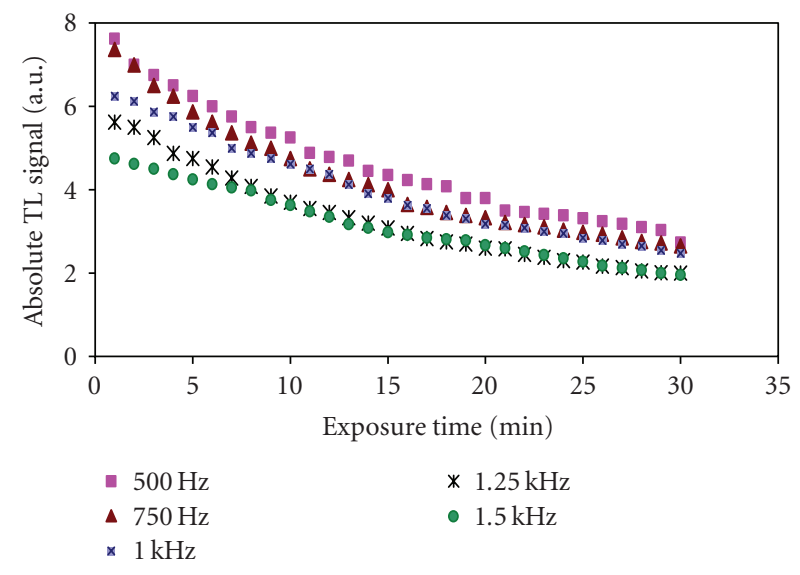

FIGURE 9: Variation of TL absolute signal of Na-fluorescein with modulation frequency. 
results clearly show that the rate of PD and TL signal amplitude of Na-fluorescein are found to increase with increasing of pump laser power and the sample concentration. The PD rate is independent of the pumping laser modulation frequency. On the contrary, the TL signal behavior has been found to be inversely proportional to the laser modulation frequency. With respect to wavelength effect, the PD rate and TL signal amplitude are found to be higher when the used wavelength is nearer to the peak absorption band of the uranine. Hence, the effect in both measurements was higher at the $488 \mathrm{~nm}$ laser wavelength than at the $514 \mathrm{~nm}$.

The photochemical quantum yield $\left(\phi_{c}\right)$ was measured using potassium ferrioxalate actinometry method for sample irradiated by argon laser of $488 \mathrm{~nm}$ wavelength. The resultant photochemical quantum yield was $2.2 \times 10^{-5}$.

\section{REFERENCES}

[1] H. de Vries, G. M. J. Beijersbergen van Henegouwen, and F. A. Huf, "Photochemical decomposition of chloramphenicol in a $0.25 \%$ eyedrop and in a therapeutic intraocular concentration," International Journal of Pharmaceutics, vol. 20, no. 3, pp. 265-271, 1984.

[2] C. O. Wilson, O. Gisvold, and R. F. Doerge, Textbook of Organic Medicinal and Pharmaceutical Chemistry, J. B. Lippincott, Philadelphia, Pa, USA, 7th edition, 1977.

[3] Merck index \& Co., "Sodium Fluorescein," C.A.S.\#: 518-47-8, Whitehouse Station, NJ, USA, 1996.

[4] J. P. Gordon, R. C. C. Leite, R. S. Moore, S. P. S. Porto, and J. R. Whinnery, "Long-transient effects in lasers with inserted liquid samples," Journal of Applied Physics, vol. 36, no. 1, pp. 3-8, 1965.

[5] R. D. Snook and R. D. Lowe, "Thermal lens spectrometry. A review," Analyst, vol. 120, pp. 2051-2068, 1995.

[6] M. Franko and C. D. Tran, "Analytical thermal lens instrumentation," Review of Scientific Instruments, vol. 67, no. 1, pp. 118, 1996.

[7] A. Kurian, N. A. George, B. Paul, V. P. N. Nampoori, and C. P. G. Vallabhan, "Studies on fluorescence efficiency and photodegradation of rhodamine 6G doped PMMA using a dual beam thermal lens technique," Laser Chemistry, vol. 20, no. 2 4, pp. 99-110, 2002.

[8] S. E. Bialkoski, Photothermal Spectroscopy Methods for Chemical Analysis, John Wiley \& Sons, New York, NY, USA, 1996.

[9] C. Jacinto, S. L. Oliveira, L. A. O. Nunes, J. D. Myers, M. J. Myers, and T. Catunda, "Normalized-lifetime thermal-lens method for the determination of luminescence quantum efficiency and thermo-optical coefficients: application to $\mathrm{Nd}^{3+}$ doped glasses," Physical Review B: Condensed Matter and Materials Physics, vol. 73, no. 12, Article ID 125107, 9 pages, 2006.

[10] G. G. Hachard and C. A. Parker, Handbook of Photochemistry, vol. 235 of Proc. Ray. Soc. A, Marcel Dekker, New York, NY, USA, 1965.

[11] S. L. Murov, Handbook of Photochemistry, chapter 13, Marcel Dekker, New York, NY, USA, 1973.

[12] J. M. Yanez-Limon, R. Mayen-Mondragon, O. MartinezFlores, et al., "Thermal diffusivity studies in edible commercial oils using thermal lens spectroscopy," Superficies y Vacio, vol. 18, no. 1, pp. 31-37, 2005.
[13] A. Philip, P. Radhakrishnan, V. P. N. Nampoori, and C. P. G. Vallabhan, "Photoacoustic study on bleaching of cresyl violet in polyvinyl alcohol by laser light," International Journal of Optoelectronics, vol. 8, no. 4, pp. 501-503, 1993. 